\title{
Dialogue and Interpretation--An Interpretive Study of the Translation of Chinese Classic Poem
}

\author{
Rongqin Ji \\ School of Foreign Languages, Jiangxi Normal University of Science and Technology, Jiangxi, China \\ ecjtu2@126.com
}

Keywords: Dialogue theory; Translation rhetoric; Translation ethics; Dialgue Interpretation

\begin{abstract}
Dialogue theory provides a new perspective on interpreting the dialogue in cultural context and communication mode in translation. Based on Bakhtin 's theory of dialogue and Robinson' s translation rhetoric and translation ethical dialogue model, this paper examines the problem how to achieve harmonious dialogue between all the parties involved in the translation of Chinese classic poem. By employing methods of exemplifications and comparison, the study made an interpretative analysis of the corpus from "A Tribute to King Teng's Tower ". It found that dialogue theory helps to avoid translation defaults such as misinterpreting and improper omission in conveying the original author's creative intention in the translation of Chinese classic poem. It suggests that dialogue model exerts certain implication on interpretation and inspiration in the translation of Chinese culture classics.
\end{abstract}

\section{Introduction}

The application of Dialogue theory to translation provides a new perspective on the translation of cultural elements and communication mode in Chinese classics. Dialogue, understanding and interpretation, constitute the fundamental mechanism of translation activities [1]. The application of dialogue theory to describe and interpret dialogue between the translator, author and the targeted reader of Chinese cultural classics, the history and reality contexts conveyed by the poem, and the communication mode of the poem, as well as to build the harmonious relationship between all the parties involved can be more clearly and evidently. Through dialogue, translators can interpret more sharply the complex relationship between the original author, the text and the targeted reader. Dialogue can also reveal more vividly and explicitly in realizing the relationship between the parties in the translation of the classics, highlighting the inter-subjectivity research of the parties and interpret them into a new vitality and more explanatory space.

\section{Translation Dialogue - Translation Rhetoric and Translation Ethics}

Translation is a process of dialogue and harmonious dance of all the parties involved. The "dialogism" in translation is based on the subjectivity in the process of translation. It offers explanation to the characteristics of dialogue in translation. Translation activities expresses two different intentions: one is the intention of the original author, the second is to reflect the translator' s intention. Robinson [2] holds that translation activity also contains two sounds, two meanings and two kinds of modality. Translation is a double-tone dialogue between the translator, the original author and the targeted reader of the translated version, which transcends time and space and transcends language and cultural barriers. It reveals the dynamics, complexity and diversity of real translation activities.

\section{Translation Rhetoric - Dialogue between Translator and the Original Author}

Robinson considers that translation rhetoric is an interpretive tool used by the translator in the dialogue with the original author. It reflects the inter functions of translation. He summarized six rhetoric devices used in the dialogue between the translator and the original author: metonymy, synecdoche, metaphor, allegory, exaggeration and double metonymy [3]. The six rhetoric images map the relationship between the translator and the original author. The translation rhetoric describes the equal relationship of cooperation between the original author and the translator. It shows the translator's respect to the original author and the pursuit tried to convey their intention. The different degree of reciprocity reflect the translator and the author's intertextual dialogue relationship. 


\section{Translation Ethics - Dialogue of Translator and Readers of the Translated Version}

Dialogue mode is the core of Robinson's translation ethics. In his view, translation is neither the monologue of the original author nor that of the translator, but rather, an open dialogue between the author of the original, the translator and the targeted reader of the translated version. Dialogue in translation is two-way rather than unidirectional, and the dialogue is carried out on an equal basis, rather than simply talking and listening. One side takes the initiative and accepts it positively. In the conversation, all the involved parties influence each other, shape each other, and hence the dialogue can change the parties [1]. Robinson summed up eight kinds of translation ethical models: introversion and extroversion, transformation and advertising, regression, subversion, distortion, transfer, diversification and dialogue. Robinson developed these on the basis of translation of body science, translation of dialogue and translation of rhetoric ethical interpretation.

\section{Dialogue Interpretation of “A Tribute to King Teng's Tower"}

"A Tribute to King Teng's Tower" was a famous classic Chinese poem. From time on, it has outgrown several English translations. To name some of them, the British Sinologist Herbert A. Giles, Henry H. Hart and so on. They are aliens translators. As this study focus on how to convey cultural elements in Chinese classics through dialogue interpretation, Chinese translators are preferred: Xiaojie Zhao and Jinguo Luo have been selected as the targeted research corpus.

The translation by Xiaojie Zhao is based on the fact that he works in the Foreign Affair office of Shangrao city, Jiangxi province. The translation is the result of his own experience as well as his own consistent research. He established an open dialogue in the translation of "A Tribute to King Teng's Tower". He merged the diachronic "visual background" into English translation, and ultimately shaped the formation of the Zhao style. He believes that "A Tribute to King Teng's Tower" belongs to Fu, a literature form near poetry. Its translation should try to achieve the effect through the maintain of long sentences. Jingguo Luo is a professor, a professional translator. He has translated lots of Chinese classic works. Contrast to Zhao, Luo prefers to take the meaning of annotation in choosing translation method.

\section{External Dialogue Rhetoric - Dialogue between the Author and the Reader}

"A Tribute to King Teng's Tower" is an outstanding representative of the parallel prose. Parallel prose style is in the syntactic structure on a dual demand, sound rhyme on the flat, rhetoric on the requirements of algae and rich use of allusion. So allusion is one of the important style of parallel prose.

For Wang Bo, it is necessary to make full use of allusion--the emotional format itself in a typical allusion factor inside, so that his personality image characteristics can consistent with the general ideal of the context. Wang Bo has the pursuit of his realistic intention and desire to meritorious career, so he choose to start from the well-known tradition of literary allusions. It is because first of all, he would like to capture the recognition of the represented guests who would might be the referee for his future development. Most of them were from the cabinet who would have such power to decide his future. He had to please the present guests one by one through rendering and flattery. Secondly, Wang Bo at that time was a nobody--with low background and lower ranks, language expression must be careful, subtle. And the rich meanings and implicit intrinsic characteristics of allusions are not only satisfies this need, but also in line with the expressions of this intention.

For the translator of "A Tribute to King Teng's Tower", he is the reader first, in the process of text interpretation, has become a translator. Bakhtin concludes the relationship between the author and the reader in the discourse as the dialogue between the "speaker" and the "listener". The speaker enters the horizons of the hearer, and in his own territory he builds his own statement on the subject's mind. The "visual background" in the dialogue field not only points to the author, but also emphasizes the author's grasp and prediction of the "visual background" of the reader, emphasizing the process of writing the text, the author and the dialogue with the reader. Allusions are mostly historical stories or historical facts, and make full use of ancient Chinese traditional culture. The Chinese traditional value distinguishes people from dignity to humble. It adheres that what happened in the past has the priority to present. This value is universally existed through Chinese history. The use of allusion is a kind of psychological strengthening and is the national character. This is more or less explained why this poem is rich in allusion. Wang Bo's target readers are a group of official travel scribes, so he consciously cited 
a lot of stories into his poem, not just rhetoric. He intentionally combined the presence of a dialogue between the readers of the presented into the historical stories. This dialogue is instant, because Wang Bo and guests are in the same era, they share a consistent "dominant background". Moreover, there is a possibility of competition between them and the possibility of flattery, that is, what Bakhtin's dialogue theory signified "dialogue priority" conditions: intercommunity. When first heard this poem, the readers were to be able to understand both the author and the readers' intentions on the basis of dialogue. Wang Bo with his majestic momentum, gorgeous rhetoric method described the magnificent and marvelous scenery, overlooked in the three autumn scene of Teng Wangge (KingTeng'sTower). At the same time, he interweaved his own depressed mood to the guests own high self-esteem state, he longed to serve the country yet without way to achieve. He intertwined all the depressed feelings with the scene, fully demonstrated the author's innerworld and feelings and mental outlook in the poem.

When the parallel prose is translated into English and entered the reader of the "other' s" vision, the background of the readers' has not been incorporated into the original writing intention of Wang Bo. In order to ensure that the readers can understand the inherited rhythmical prose of Tang Dynasty and through the rich stories in the allusion, not only can read out the specific historical context of the development of China's humanistic spirit, but also can grasp the spirit through the allusion of the process of the good inherited development of literature and art reference, as well as its outspread meaning, smooth deformation of the art Characteristics. To achieve such an intention, the translator must rely on the background of the introduction to make up for the present readers' "previous understanding" of the missing, or rather, it would be difficult for them to appreciate the beauty of the ancient poem as well as the internalized moral values of the poet and his ideal character of personality. If the readers of the time is not the official travel scholar, the general reader is difficult to understand the meaning of this poem, but simply understood as a landscape description of the lyrics, such as the direct citation of the ancient metaphor and the overt use of "its products are nature's jewels. The radiance of its legendary sword shoots directly upward between the constellations Niu and Dou". When the allusion is translated into English, the cultural background is completely different to the Western readers. Their "visual background" determines that they can not establish a dialogue with the original author of the poem. The replace of the original author to communicate with them has to be the translator. While translators, like the original author, also need to figure out the reader's "visual background" first -- he has to establish the dialogue with the author first to enable the translation go smoothly. But at this time, the translator and the targeted readers do not share the same "visual background" as that with Wang Bo. They can not experience the severe competition relationship and the flattery between the official travels and each other. Even if the translator himself can figure out the allusion of Wang Bo's intention, to fully reflect the deeper intention of this allusion is still very difficult, as there are no stories like "Glorious Dragon light" and "Couch" in the western readers" "visual background". Here "dragon light" refers to the light reflected from the sword, which is from the legend of the cattle from Jin dynasty. It is said that fighting between the two stars often appear purple light, which in the eyes of the ancients is undoubtedly a heaven-sent Rui Zhao (propitious sign). So Zhang Hua of Jin Dynasty invited Nanchang people Lei Huan to have a look. Lei Huan said it was the light of the sword on the sky. Soon it happened that in the fortress of Feng Cheng's underground there were really two swords unearthed, and the sky then gradually dissipated the purple. This allusion contains the traditional Chinese culture in the worship to heaven, a kind of worship to supernature. All the things in the world have spirit, spirit is eternal. The western reads here may associate with the western astrology theory, but astrology, after all, is linked to religion and witchcraft, while China's spirit concept can not completely be analogized. The term "constellation" is the most appropriate explanation to the Chinese concept of stars in English. "Couch" was a story of the Eastern Han Dynasty. It says that Xu Ruzi was a knowledgeable person but indifferent to fame and fortune. His high valued behavior was widely admired by people. The story of Chen Fan talks that when Chen Fan was the governor of Yuzhang County behaved very elegantly. When official Li Yin came, Xu Ruzi specifically set up a couch for Li Yin. When he came, Xu Ruzi put down the coach, while when he left, the coach was immediately hang up. This became a good story to show Xu Ruzi's scrupulously behave. 
It is impossible for the translator to take this into account when he tries to figure out the background of the writer. Since the translator is in a special position to dialogue with both the original writer and the targeted reader, it is hard for him to strike a balance between the original writer and the reader of the translated version. So:

Luo: The radiance of its legendary sword shoots directly upward between the constellations Niu and Dou. Its talented people are outstanding, and the spirit of intelligence pervades the place. This was the place where Xu Ru spent the night on his visit to Chen Fan [4].

Zhao : With God blessing rich and delicious products, swords' light shining towards the constellation of Niu and Dou Stars' space. Full of talents and land is intelligent, Chenfan specially set up a part-time bed for Xuru [5].

Both Luo and zhao chose free translation with annotation method in the translation of allusions. In this way, Luo did not mention the word "couch", but simply said, "This was the place where Xu Ru spent the night on his visit to Chen Fan", and then noted that Chen Fan and Xu Ruzi's identity and story. He extracted the original part of the most representative of the translation, to seize the core content, to abandon the minutiae, only to convey the original meaning. This has not only alleviated the difficulty of Western readers to understand, but also increased the fun of reading. It is highly meet with Robinson's "metaphor translation" rhetoric requirements. While Zhao's version clearly add more interpretations to the original. Zhao has fully take the difficult "dominant background" of the western readers into consideration, and almost satisfied the requirements of "metaphor translation". The purpose to construct a harmonious dialogue between the author, the translator and the targeted reader thus have achieved.

\section{Internal Dialogue - Dialogue between the Author and Social Consciousness}

The key to the translation of “A Tribute to King Teng's Tower" lies not only in its rich rhetoric, but also in the construction of allusion, and the passing of the profound and enigmatic thoughts. How to convey the cynical feeling through the description of lyric scene, the inner feelings of national portrait in "A Tribute to King Teng's Tower", is a topic related to "dialogue". How to establish the dialogue is not only in accordance with the author's historical background, with his social identity, but also with his distinctive personality. The potential lies to the multi-relationship in the dialogue, which is just as what Bakhtin's monologue states. Bakhtin argues that there is also a dialogue between "self" and "the other" in monologue discourse, where "the other" can be either a general sense of society or a subjective consciousness of objectivity. The hero is a image of thought and a contradictory body, and because the author "takes an objective world to compete with the omnipotent consciousness of the hero, this objective world is the world of many others who are aware of equality" [6]. Wang Bo in "A Tribute to King Teng's Tower" takes the opportunity to express the noble spirit in the chest, real life in the virtual, seeing God in tangible. These directly reflect his personal consciousness. Yet it overly implies that this parallel prose represented by a variety existence of social consciousness. In fact, in addition to the protagonist consciousness, the whole poem, from the beginning to the end, exist a general sense of social consciousness. This consciousness conveys the social status, power and other factors on personal emotional constraints. Wang Bo not only depicting the poem around the scenery and feast grand occasion, but put more emphasis on the end of feeling: life encounter, self-expression world, infinite indignation, overflowing on the paper. From the poem with the code of the ideological content of the view, he issued a sincere sigh: "Alas! I am ill fated, and my life is full of frustrations" . Wang Bo has suffered two major career life setbacks, talent and aspirations. They made him withstand an unprecedented experience and toughening. All kinds of frustration Wang Bo encountered made him in the poem subconsciously sustenance more profound thinking and life accumulation. Allusions cited in "A Tribute to King Teng's Tower" have apparently inherited the literary tradition of the Six Dynasties, reflecting the author's subjective consciousness, his strong desire to get the identity of the aristocratic social identity, so as to re-establish his own social reality in an ideal position, and to achieve ambitious social ambitions. This is the historical conditions of a social voice, reflecting a common sense of the common role of society. When the author is in the congregation, it is in the general consciousness of the dialogue. But the ideal and the reality of the contradiction of the individual intelligence and social position is not always quite a recognized performance in the poem. The allusion in the creation, in the 
preface with the performance is also very prominent. In translation, because the translator is faced with the general collision of social differences, individual and social consciousness, the diction of the poem must also be different, which directly affects the translator in decision. As a reference, we can compare the the following translation:

Luo: The sky is high and the land is boundless; I cannot but feel the immensity of the universe. Sadness follows happiness. I am aware that success and failure are predestined. [4]

Zhao: Endless sky and boundless land, universe is inexhaustible. Bliss passes, and sadness will come, understand the rise and decline are decreed by fate. [5]

Luo directly used the first person "I" in the translation, highlighting the author's hero role, as well as the overt expression to show Wang Bo's political ambitions. It depicts the author's lament of his depressed life, difficulty in achieving the ideal, unhappy mood of loss and melancholy, the mood of the feelings of love are all evidenced between the lines. However, Zhao's translation does not take the way as Luo, he does not use the first person "I", not directly highlighting the author's hero role, rather, he intentionally omitted the author's presence. Though he gave hints to the author's presence, he chose word-for-word translation. This way, to modern readers, is hard to understand and experience the author's depress feeling and his strong desire to get the identity of the aristocratic social identity, so as to re-establish his own social reality in an ideal position, and to achieve ambitious social ambitions. Thus, the failure to convey this dialogue relationship between the author and the social consciousnesses could cause deficiency in the understanding of the targeted readers.

\section{Conclusion}

From translation rhetoric to translation ethics, from "double-tone dialogue" to monologue dialogue, from the formal dialogue to the spirit dialogue, dialogue gives people external position and space-time structure, which are full of participatory spirit and thinking. The translation is neither the monologue of the original author nor the monologue of the translator, but an open dialogue that the original author, the translator and the targeted reader participate in the translator's physical and mental experience. Through dialogue, the translator and the author are unified to reveal the nature of the discourse philosophy of the translation of classics, to explore the construction of the intertextuality to the meaning of the diachronic text. Through dialogue, translation of Chinese classics can inherit and carry forward the cultural heritage of the Chinese culture, enhance the national self-confidence in the communication with the others.

\section{Acknowledgments}

This research has been sponsored by the project of the Doctoral Fund of Jiangxi Science and Technology Normal University (2015). It is a partial achievement of the Social Science Research Project issued by Jiangxi Provincial Association of Social Science (15YY21).

\section{References}

[1] Z. J. Wang. Translation on the Perspective of Dialogue Theory, Journal of Chongqin Jiaotong University, (2009), No.5, P23.

[2] R. Douglas. The Translator's Turn (Baltimore: Johns Hopkins University Press, United States 1991) p.56

[3] L. N. Wang . Translation: a double dialogue - analysis of Douglas Robinson's Dialogue Theory, Journal of Foreign Language, (2009), No. 3, p33-36.

[4] J. G. Luo. Translated. The Finest of Ancient Prose (Beijing: Foreign Language Teaching and Research Press, Beijing 2005) p 35. 
[5] X. J. Zhao. A Tribute to King Teng's Tower, Journal of Jiangxi Education Institute, (2009), No. 1, p39.

[6] Bakhtin, Mikhail. Problems of Dostoevshy's Poetics (Caryl Emerson (ed.). Minneapolis: University of Texas Press, United States 1984) p13. 